\title{
Wer kennt wen und was geht ab?
}

\author{
Ein netzwerkanalytischer Blick auf die Rolle delinquenter Peers \\ im Rahmen der Situational Action Theory
}

von Dominik Gerstner und Dietrich Oberwittler

\section{Zusammenfassung}

Eine der zentralen Annahmen der Forschung zur Jugenddelinquenz ist, dass Jugendliche besonders dann Straftaten begehen, wenn sie sich in Begleitung anderer Jugendlicher und fernab der Aufsicht durch Erwachsene befinden. Belege hierfür finden sich auch in neueren Studien, die auf dem Theoriegerüst der »Situational Action Theory (SAT) aufbauen. Die SAT nimmt an, dass während gemeinsamer Freizeit mit zur Delinquenz neigenden Freunden Tatgelegenheiten und Risiken verstärkt werden - dies jedoch in Abhängigkeit individuell sehr unterschiedlicher devianter Neigungen. Die Delinquenz von Freunden ist in empirischen Untersuchungen besonders schwer zu messen, und in der aktuellen Forschung finden sich hierzu interessante Ansätze. Verlässt man sich, wie traditionell üblich, auf Angaben, die Befragte über ihre Freunde machen (indirekte Messung), so wird die eigene Delinquenz häufig auf die Delinquenz der Freunde projiziert und damit der Zusammenhang zwischen eigener Delinquenz und der der Freunde überschätzt. Eine Alternative stellt die direkte Messung der Delinquenz der Freunde dar, die in der Regel ein realistischeres Bild wiedergibt. Dabei sind die Freunde über Netzwerkdaten mit den Befragten verbunden und geben selbst Auskunft über ihr Verhalten.

In unserer Untersuchung vergleichen wir anhand neuer Daten einer Schulbefragung die direkte und indirekte Messung der Delinquenz der Freunde im Hinblick auf ihre Rolle bei der Erklärung von Delinquenz im Rahmen der SAT. Es zeigt sich, dass die indirekte Messung den Einfluss von Situationen und Gelegenheiten verdeckt. So zählt in multivariaten Modellen für die Vorhersage der eigenen Delinquenz lediglich, welche persönlichen Neigungen und welche Freunde Jugendliche haben. Bei einer direkten Messung zählt darüber hinaus auch, ob man mit diesen Freunden einen risikoreichen Freizeitstil teilt.

Schlüsselwörter: Situational Action Theory, SAT, Netzwerkanalyse, delinquente Peers, negative Binomialregression, Interaktionseffekte

\section{Who's hanging out and what's going on?}

A network perspective on delinquent peers in the framework of Situational Action Theory

\section{Abstract}

One of the key observations of delinquency research - that adolescents are more likely to offend during the time spent in the company of peers and without adult supervision - has been supported by recent studies following the Situational Action Theory (SAT) framework. According to SAT, spending time with delinquent friends may influence adolescent behavior by altering opportunities and frictions in certain settings, but at the same time the effect of these situational factors is dependent on individual crime propensities which vary between adolescents.

One issue of continuing interest in recent research is the measurement of delinquent friends. The association between a person's own delinquency and his friends' delinquency is overestimated when respondents report about their friends' behavior (indirect measurement). Studies using social network analysis to construct a direct measure of delinquent friends based on the friends' self-reported delinquency tend to give a more realistic picture of this association. In this study, we use data from a recent German school survey including network data and a direct measurement of delinquent friends. We analyze how the role of delinquent friends and the effects of a risky lifestyle in explanatory models within the SAT framework change if one moves from an indirect to a direct measurement of friends' delinquency. It can be shown that the influence of situations and opportunities may be obscured when using an indirect measurement and the prediction of delinquency only depends on who you are and who your 
friends are. When using a direct measure it is also important whether the individual shares a risky lifestyle with his or her friends.

Keywords: Situational Action Theory, network analysis, delinquent peers, negative binomial regression, interaction effects

\section{Einleitung}

Eine der maßgeblichen Ursachenkomplexe bei der Erforschung der Delinquenz von Jugendlichen waren seit Beginn die Gleichaltrigenbeziehungen. Schon früh verwiesen Forscher wie z.B. Shaw E McKay (1931) auf den Gemeinschaftscharakter von Straftaten im Jugendalter, und heute gilt die gefestigte Annahme, dass Jugendliche oft dann Straftaten begehen, wenn sie sich in Begleitung anderer Jugendlicher außerhalb des Bereichs elterlicher Kontrolle befinden (vgl. z.B. Warr 1996). Die kausalen Mechanismen, durch die die Gleichaltrigenbeziehungen auf delinquentes Verhalten wirken, sind ohne Zweifel komplex. Man kann in der Theoriebildung zwei Hauptrichtungen unterscheiden (vgl. Haynie E Osgood 2005; Thomas $\varepsilon$ McGloin 2013): Eine historisch ältere Richtung sieht den normativen Einfluss der Gleichaltrigen auf Einstellungen und Handlungsdispositionen von Jugendlichen als maßgeblich an. Dies gilt für die »klassischen « Lern- und Subkulturtheorien (Burgess E Akers 1966; Akers E Jensen 2003). Eine andere, noch relativ junge Richtung hebt eher den Effekt der Gelegenheiten auf die Wahrscheinlichkeit von Delinquenz hervor, die sich durch die gemeinsame (Frei-)Zeit mit Gleichaltrigen erhöht. Hier spielt also der situative Kontext delinquenten Handelns die zentrale Rolle. Auftrieb erhielt dieses Erklärungsmuster durch den »Routine Activity Approach «(Cohen E Felson 1979), auch wenn bereits Sutherland (1947, zitiert bei Thomas E McGloin 2013, 436) und vor mehr als 150 Jahren Quetelet (1921 [orig. 1835], 252) die Bedeutung situativer Faktoren erkannt hatten. Wayne Osgood war einer der ersten Kriminologen, der mit seinen Kollegen empirische Belege für die eigenständige Rolle von Gelegenheiten in Form alterstypischen Freizeitverhaltens auf Jugenddelinquenz vorgelegt hat (z.B. Osgood et al. 1996; Hughes E Short 2014).

Die Frage, welche von beiden Wirkmechanismen (oder auch welche anderen Einflüsse) für die Entstehung von Delinquenz maßgeblich sind, muss nicht mit einem exklusiven »Entweder-Oder « beantwortet werden. Im Gegensatz zu den »großen « Theorieansätzen mit hohem Allgemeingültigkeitsanspruch, wie z.B. die Kontrolltheorie (Hirschi 1969) und die General Theory of Crime (Gottfredson E Hirschi 1990), hat sich die aktuelle Forschung in den letzten Jahren verstärkt integrativen Erklärungsansätzen zugewendet. Dabei geht man zunehmend davon aus, dass unterschiedliche Einflüsse nicht nur additiv, sondern auch multiplikativ auf delinquentes Verhalten wirken (Oberwittler 2015). Multiplikativ bedeutet, dass Einflussfaktoren nicht unabhängig voneinander jeweils für sich wirken - und somit das eine zum anderen kommt -, sondern dass zwischen den Einflussfaktoren Wechselwirkungen (Interaktionen) bestehen, d.h. die Wirkung des einen Faktors hängt von der Ausprägung eines anderen $a b$, oder zwei Faktoren verstärken sich gegenseitig. In Bezug auf die Bedeutung der Gleichaltrigenbeziehungen für Delinquenz stellt sich beispielsweise die Frage, von welchen weiteren Faktoren es abhängt, welche Effekte Freundschaften mit anderen delinquenten Jugendlichen haben oder unter welchen Umständen gemeinsam verbrachte Freizeit das Delinquenzrisiko steigert (Hoeben E Weerman 2014; Svensson E Oberwittler 2010; Weerman et al. 2013).

Als neuer, integrativer Theorieansatz, der die Rolle situativer Einflüsse für die Entstehung von Delinquenz in den Mittelpunkt rückt und dabei zudem die Wechselwirkungen unterschiedlicher personaler und situativer Faktoren explizit berücksichtigt, eignet sich die Situational Action Theory von Wikström $(2004 ; 2010)$ besonders für die differenzierte Untersu- 
chung der Auswirkungen der Gleichaltrigenbeziehungen. In den hier vorgestellten Analysen greifen wir wichtige Grundannahmen der SAT auf und nutzen sie für eine empirische Analyse auf der Basis der Daten der Schulbefragung »Lebenslagen und Risiken von Jugendlichen« (vgl. Oberwittler, Schwarzenbach E Gerstner 2014).

In Studien zur Jugenddelinquenz auf der Basis von Befragungsdaten stellt die Art der Messung von Peer-Delinquenz ein wichtiges Problem dar. In einigen Studien konnte gezeigt werden, dass die konventionelle, »indirekte "Messung, die sich auf die Wahrnehmung der Befragten bezüglich der Delinquenz ihrer Freunde verlässt, zu einer Überschätzung des Einflusses delinquenter Freunde und einer Beeinträchtigung anderer Einflussgrößen tendiert (Haynie 2001; Haynie E Osgood 2005; Weerman E Smeenk 2005; Meldrum, Young E Weerman 2009). Mit der Verwendung von Netzwerkdaten kann die Peer-Delinquenz direkt gemessen werden, was zu einer Verbesserung der Erklärungsmodelle beiträgt. Auch diesen Aspekt wollen wir in den empirischen Analysen berücksichtigen.

\section{Situational Action Theory und delinquente Peers}

Mit der Situational Action Theory (im Folgenden SAT) fasst Wikström $(2004 ; 2010)$ das interaktive Zusammenspiel unterschiedlicher Theorietraditionen in der Analyse von Jugenddelinquenz in einen gefestigten Rahmen. Die Grundidee der SAT wird in der scheinbar einfachen Frage ausgedrückt, wer sich in welcher Situation befindet. Delinquentes Verhalten resultiert dann aus dem Zusammenspiel beider Komponenten, also persönlicher und situativer Merkmale. Die delinquente Handlung wird als Ergebnis eines » Wahrnehmungs-Entscheidungs-Prozesses « (perception choice process, im Folgenden PCP) bezeichnet, der in der Literatur mit der Formel $\mathbf{P} \times \mathbf{E} \rightarrow \mathbf{C}$ symbolisiert wird. Dabei interagieren Individuen, die mit bestimmten Neigungen zur Delinquenz (crime propensity $\mathbf{P}$ ) ausgestattet sind, mit ihrer physischen und sozialen Umwelt (exposure to criminogenic settings E). Je nach Handlungsentscheidungen ergibt sich in den jeweiligen Situationen delinquentes oder auch nicht-delinquentes Verhalten. Eine ausführliche Zusammenfassung und detaillierte empirische Überprüfung der Theorie wurde unlängst mit der Monographie »Breaking Rules« (Wikström et al. 2012) vorgelegt. Auch andere Forscher haben die SAT in ihren Studien bereits empirisch getestet (z.B. Svensson, Pauwels E Weerman 2010; Wikström E Svensson 2010; Beier 2014).

Für den vorliegenden Beitrag ist die Frage nach dem Wer von besonderer Bedeutung. Zum einen geht es um einzelne Individuen, welche moralische oder auch strafrechtliche Grenzen überschreiten. Zum anderen geht es darum, mit wem diese Individuen ihre Zeit verbringen und welchen Aktivitäten dabei nachgegangen wird. Dabei soll untersucht werden, welchen Einfluss delinquente Freunde haben und wie diese innerhalb des PCP verortet werden können.

Die Neigung zu delinquentem Verhalten (crime propensity $\mathbf{P}$ ) definiert sich darüber, dass Individuen Verhaltensregeln und Normen unterschiedlich internalisieren und sich mehr oder weniger stark an diese halten. Dies hängt von moralischen Überzeugungen und Schamempfinden, aber auch der Fähigkeit zur Selbstkontrolle ab, welche zusammen bereits einen großen Teil der Delinquenz von Jugendlichen erklären können. Dieser »moralische Filter « wirkt jedoch nicht immer gleich, sondern interagiert mit bestimmten Situationen, wie z.B. dem Freizeitverhalten von Jugendlichen. Die SAT bezeichnet dies als »exposure to criminogenic settings « $(\mathbf{E})$, in denen z.B. Verlockungen zur Begehung von Diebstählen oder Anlässe zu Gewalthandlungen unterschiedlich stark ausgeprägt sein können. Es wird dabei angenommen, dass die kriminogenen Situationen im Mittel einen eigenen kriminalitätsfördernden Effekt haben, der aber je nach individueller Neigung stärker oder schwächer ausfällt bzw. auch gar nicht vorhanden ist. 
Mit dem situativen Kontext wird auch die Rolle der delinquenten Peers relevant. Einer der zentralen Befunde der Erforschung abweichenden Verhaltens Jugendlicher ist, dass Jugendliche mit delinquenten Freunden eher selbst delinquent sind oder werden (z.B. Elliott, Huizinga E Ageton 1985; Warr 2002; Haynie E Osgood 2005). Dieser Zusammenhang kann zum einen als Folge eines Selektionseffekts betrachtet werden, nach dem delinquente Jugendliche bevorzugt Freundschaften mit anderen delinquenten Jugendlichen eingehen (z.B. Hirschi 1969). Zum anderen kann man annehmen, dass Jugendliche erst durch den Einfluss delinquenter Freunde selbst delinquent werden, was dem Ansatz von klassischen Lerntheorien entspricht (z.B. Sutherland 1947; Akers 1973). In der neueren Forschung wird - unterstützt von Längsschnittstudien - davon ausgegangen, dass beide Annahmen zutreffend sind (z.B. Thornberry et al. 1994; Steglich, Snijders E Pearson 2010; Svensson et al. 2012). Unabhängig von der Kausalrichtung ist relevant, dass durch delinquente Peers auch die Tatgelegenheiten erhöht werden, denn - wie bereits erwähnt - begehen Jugendliche vor allem dann Straftaten, wenn sie mit anderen Jugendlichen zusammen und fernab der Kontrolle von Eltern oder anderen Erwachsenen sind und unstrukturierten Freizeitaktivitäten nachgehen. In diesen Situationen treffen im Sinne von Cohen E Felson (1979) potentielle Täter vermehrt auf Tatgelegenheiten, und die mögliche Tatbegehung wird zudem durch reduzierte soziale Kontrolle erleichtert. Außerdem werden Motivationen auch dadurch verstärkt, dass abweichendes Verhalten von Gleichaltrigen honoriert wird und Jugendliche in der Gruppe höhere Risiken eingehen (z.B. Osgood et al. 1996; Gardner E Steinberg 2005). Dies führt zu der empirisch bereits bestätigten Annahme, dass mit zunehmender Delinquenz der Freunde ein höheres delinquentes Verhalten einhergeht (vgl. Warr 2002). Studien zur SAT haben diese Tatsache bisher nur am Rande untersucht. Bei Wikström et al. (2012, 151 ff.) wird zwar darauf hingewiesen, dass die Delinquenz der Freunde den Effekt der »criminogenic exposure « moderiert, dies wird jedoch nur als Beleg dafür herangezogen, dass das delinquente Verhalten der Freunde ein Indikator für deren kriminelle Neigungen sei. Letztendlich werden aber beide Maße zu einem Konstrukt verbunden und im Zusammenhang mit den persönlichen kriminellen Neigungen nicht getrennt betrachtet. Auch in anderen Publikationen zur SATwerden Peer-Delinquenz und unterschiedliche Messungen zu unstrukturierten Freizeitaktivitäten als ein Konstrukt gesehen (Wikström E Svensson 2008; Wikström 2009; Svensson E Pauwels 2010). Die Frage, was man mit wem macht, wird dabei im Wesentlichen ignoriert. Untersucht wird dies jedoch bei Svensson E Oberwittler (2010), die zeigen können, dass die Delinquenz der Freunde den Effekt der unstrukturierten Freizeitaktivitäten moderiert (Gleiches gilt auch in umgekehrter Richtung). Die individuellen Neigungen spielen aber in dieser Studie nur bedingt eine Rolle. So bleibt die Frage bestehen, wie sich das Zusammenspiel zwischen individuellen Neigungen und kriminogenen Gelegenheiten ändert, wenn die Delinquenz der Freunde als eigenständige Komponente analysiert wird. Es ist davon auszugehen, dass dieses für Jugendliche mit vielen delinquenten Freunden ausgeprägter ist, da zu den vermehrten Gelegenheiten hinzukommt, dass der Einzelne in Begleitung delinquenter Peers ist. Dies hat wiederum einen steigernden Effekt auf die eigene Delinquenz bzw. die kriminogene Situation. Wir formulieren aufgrund unserer Ausführungen die folgenden Hypothesen:

H1: Mit höherer individueller Neigung zur Kriminalität gehen mehr begangene Straftaten einher.

H2: Die Wahrscheinlichkeit von Straftaten steigt, wenn Jugendliche ihre Freizeit mit Gleichaltrigen in unstrukturierten Freizeitaktivitäten fernab elterlicher Kontrolle verbringen und damit mehr potentiell kriminogenen Situationen ausgesetzt sind. 
H3: Zwischen individueller Neigung und kriminogenen Situationen besteht eine Interaktion. Kriminogene Situationen münden seltener bzw. gar nicht in Straftaten, wenn Jugendliche eine geringe bzw. keine Neigung zu kriminellem Verhalten aufweisen.

H4: Der Umgang mit delinquenten Peers geht mit höherer eigener Delinquenz einher.

H5: Abhängig von der Delinquenz der Peers fällt die Interaktion zwischen individueller Neigung und kriminogener Situation unterschiedlich aus (3-Wege-Interaktion). Je delinquenter die Peers, desto ausgeprägter die Interaktion zwischen individueller Neigung und kriminogenen Gelegenheiten.

\section{Zur Messung delinquenter Peers}

Im Zusammenhang mit Befragungsdaten ist seit längerem bekannt, dass Befragte nur begrenzte Fähigkeiten haben, über das Verhalten und die Einstellungen anderer Personen Auskunft zu geben (z.B. Jussim E Osgood 1989; Aseltine 1995). Dies trifft insbesondere auf Angaben zur Delinquenz von Freunden zu, die in den meisten kriminologischen Studien über die von Elliott et al. (1983) eingeführte Messung erfolgt, bei der die Befragten selbst Auskunft über die Delinquenz ihrer Freunde geben. Diese wird dabei häufig dahingehend verzerrt eingeschätzt, dass die eigene Delinquenz auf die Delinquenz der Freunde projiziert wird. Gottfredson und Hirschi vermuten in diesem Zusammenhang sogar, dass damit lediglich die eigene Delinquenz gemessen wird: »[. . .] the variable - self-reported peer delinquency - may merely be another measure of self-reported delinquency « Gottfredson E Hirschi 1990, 157). Inwieweit dies zutrifft und welche Folgen sich hieraus ergeben, wurde mit dem Einsatz von Netzwerkdaten in neueren Studien empirisch überprüft. Haynie E Osgood (2005) konnten z.B. nachweisen, dass der Zusammenhang zwischen eigener Delinquenz und Peer-Delinquenz unter Verwendung einer direkten Messung durch Netzwerkdaten deutlich geringer ausfällt, und die Überschätzung des Einflusses von Peer-Delinquenz durch die konventionelle und indirekte Messung zur Unterschätzung anderer Einflussfaktoren geführt hat. Zu ähnlichen Ergebnissen kommen auch Meldrum et al. (2009), die den Einfluss von Selbstkontrolle unter Kontrolle von indirekter und direkter Messung der Peer-Delinquenz vergleichen. Die gleiche Gruppe von Autoren kommt auch zu dem Schluss, dass dieaus der Differenz von direkter und indirekter Messung errechnete - Fehlwahrnehmung hauptsächlich durch die eigene Delinquenz und das Konzept der Selbstkontrolle erklärt werden kann (Young et al. 2011). Ausgehend von diesen Beobachtungen kann bezweifelt werden, dass die Rolle der delinquenten Freunde innerhalb der SAT empirisch ausreichend geprüft wird, wenn lediglich eine indirekte Messung der Peer-Delinquenz vorliegt. Die uns vorliegenden Daten lassen einen Vergleich von direkter und indirekter Messung zu, und es soll diesbezüglich folgende Hypothese geprüft werden:

H6: Unterschiedliche Messungen von Peer-Delinquenz wirken sich verschieden auf abhängige und unabhängige Variablen aus. Durch die Fehlwahrnehmung wird der Zusammenhang zwischen der Delinquenz der Freunde und der eigenen Delinquenz stark überschätzt. Gleichzeitig wird durch diesen speziellen Messfehler der Einfluss der unstrukturierten Freizeittätigkeiten mit anderen Jugendlichen überstrahlt. Dies führt zu möglicherweise falschen Schlüssen bezüglich der Rolle delinquenter Peers innerhalb der SAT.

\section{Daten und Analysestrategie}

Die für unsere empirische Untersuchung herangezogenen Daten entstammen der Schulbefragung »Lebenslagen und Risiken von Jugendlichen «, die von September 2011 bis März 2012 in den deutschen Großstädten Köln und Mannheim vom Max-Planck-Institut für aus- 
ländisches und internationales Strafrecht Freiburg durchgeführt wurde. ${ }^{1}$ In beiden Städten haben insgesamt ca. 7.300 Schülerinnen und Schüler ${ }^{2}$ an 63 allgemeinbildenden Schulen an der Befragungsaktion teilgenommen. Die Jugendlichen besuchten während der Befragung die Jahrgangsstufen 8 bis 10. Eine Ausnahme stellen einige wenige Schüler aus Mannheim dar, die ihren Realschulabschluss an einer Berufsfachschule nachholen.

Der verwendete Fragebogen enthielt Fragen zum soziodemografischen Hintergrund der Schüler, der familiären Situation und dem sozialen und schulischen Umfeld. Zudem wurden Einstellungen zur Delinquenz und Erfahrungen mit delinquentem Verhalten von den Schülern selbst sowie auch ihren Mitschülern und Freunden erfragt. Einen weiteren und für unsere Untersuchung zentralen Teil stellen außerdem die Freundschaftsnetzwerke innerhalb der Klasse dar, die ebenfalls durch den Fragebogen erhoben wurden. Zu Beginn der Befragung wurde eine Klassenliste an die Wand projiziert, über welche den Schülern ein eigener anonymer Code zugewiesen wurde. In die Netzwerkmatrix auf dem Fragebogen mussten die Schüler nun zum einen ihren eigenen Code eintragen, zum anderen konnten durch Eintragen der entsprechenden Codes bis zu acht Mitschüler aus derselben Klasse als Freunde nominiert werden. Zu jeder Freundschaft wurden Attribute zu gemeinsamer Freizeit, Enge und Dauer der Freundschaft abgefragt. Aus diesen Angaben kann nun beispielsweise über die selbstberichtete Delinquenz der Freunde ein alternatives Maß der Delinquenz der Freunde berechnet werden.

Zu den Vor- und Nachteilen unterschiedlicher Erhebungen von Netzwerkdaten (z.B. Vorgabe der Anzahl der Nominierungen) bestehen in der Literatur unterschiedliche Meinungen (vgl. Cillessen 2009; Kossinets 2006), die hier nicht diskutiert werden sollen. Ein dringlicheres Problem stellen fehlende Netzwerkknoten dar, die durch eine geringe Ausschöpfung einzelner Schulklassen zustande kommen. Aufgrund ihrer relationalen und komplexen Struktur sind Netzwerkdaten besonders empfindlich gegenüber fehlenden Werten (vgl. Burt 1987), was auch in unserer Untersuchung nicht vernachlässigt werden kann. Während z.B. der Unit-Nonresponse bei Befragungsdaten unabhängiger Einheiten - sofern MCAR - meist nur die Fallzahl reduziert, wirkt sich dieser bei Netzwerkdaten auch auf die übrigen Einheiten aus. In unserem Fall kann z.B. die durchschnittliche Delinquenz der befreundeten Klassenkameraden eines Schülers nicht korrekt berechnet werden, wenn dessen Freunde nur teilweise an der Befragung teilgenommen haben.

Die Frage des Umgangs mit fehlenden Werten bei Netzwerkdaten hängt auch von den Analysemethoden (Kossinets 2006) und der Frage ab, inwieweit die fehlenden Werte die Knoten- und/oder Kantenvollständigkeit betreffen (vgl. Erlhofer 2008). In manchen Fällen ist es möglich, fehlende Werte auf verschiedenen Ebenen zu imputieren (vgl. Handcock E Gile 2007). Bezogen auf die Knotenunvollständigkeit, in unserem Fall fehlende Schüler, ist es aber durchaus auch zulässig, eine gewisse Anzahl an fehlenden Werten zu tolerieren. In Simulationsstudien konnte gezeigt werden, dass gängige Netzwerkmaße bis zu einer Anzahl

1 Die Schulbefragung wurde im Rahmen des Projekts »Police and Adolescents in Multi-Ethnic Societies « (POLIS) durchgeführt. Das Projekt wurde mit Mitteln des von der Deutschen Forschungsgemeinschaft (DFG) und der Agence Nationale de la Recherche (ANR) getragenen »Förderprogramms in den Geistes- und Sozialwissenschaften 2008 « gefördert. Die Leiter des deutschen Teilprojekts waren Hans-Jörg Albrecht und Dietrich Oberwittler (Max-Planck-Institut für ausländisches und internationales Strafrecht, Freiburg), der Leiter des französischen Teilprojekts war Sebastian Roché (Universität Grenoble). In Köln wurde die Befragung vom Max-Planck-Institut Freiburg gemeinsam mit dem Forschungsinstitut für Soziologie an der Universität Köln durchgeführt. Die Befragung wurde von den Schulaufsichtsbehörden und den beiden Städten unterstützt. Siehe hierzu auch https://jugendforschung.mpicc.de/de/startseite.html [15.12.2014].

2 Im Weiteren wird aus sprachlichen Gründen der männliche Begriff "Schüler « als geschlechtsneutraler Ausdruck verwendet. 
von $10 \%$ fehlender Knoten relativ robust bleiben (Borgatti, Carley E Krackhardt 2006). In einer anderen Studie wurde mit Netzwerkdaten aus Schulklassen untersucht, inwieweit sich das zufällige Entfernen von Netzwerkknoten auf die Klassifizierung (beliebt, unbeliebt etc.) von Schülern auswirkt. Eine signifikante Abnahme der Korrektheit der Klassifizierung war ab $20 \%$ fehlender Knoten zu beobachten (vgl. Crick E Ladd 1989).

\subsection{Klassennetzwerke und Freundschaften in Schulklassen}

Aus den Schulbefragungsdaten ergibt sich folgende Situation: Der Gesamtrücklauf der Befragung liegt bei ca. $75 \%$. Die Rücklaufquote variiert dabei zwischen den Klassen deutlich. Von insgesamt 373 befragten Klassen wurden für 335 Klassen Netzwerkdaten erhoben. Neben dem vollständigen Antwortausfall ist für die Vollständigkeit der Netzwerke auch relevant, ob die Schüler ihre eigene ID angegeben haben. In manchen Fällen wurde dies vergessen oder aber auch explizit verweigert. Für die vorliegenden Analysen wurden nur vollständige Klassennetzwerke analysiert. Als vollständig gelten Klassen für uns dann, wenn maximal 10 \% (bzw. 15 \% bei sehr großen Klassen) der Knoten fehlen. Die Gesamtstichprobe von 7.296 Schülern in 373 Klassen reduziert sich dabei auf eine Teilstichprobe von 3.083 Schülern in 127 Klassen. Die Teilstichprobe weicht in ihrer Charakteristik von der Gesamtstichprobe insofern ab, dass beispielsweise Gymnasialklassen deutlich überrepräsentiert sind. Dieser Aspekt soll in der Schlussbetrachtung nochmals aufgegriffen werden.

Neben der Frage nach fehlenden Werten stellt sich auch die Frage der Grenzen von Netzwerken. Freundschaftsnetzwerke in Schulklassen stellen einen relativ eng begrenzten Rahmen dar, der je nach Fragestellung zu mehr oder weniger validen Ergebnissen führen kann (vgl. Friemel E Knecht 2009). Bei Längsschnittanalysen mit dynamischen Netzwerken hat sich beispielsweise herausgestellt, dass der Einfluss der Delinquenz von Freunden keinen signifikanten Effekt auf die eigene Delinquenz hat, wenn das Netzwerk aus Klassenkameraden besteht. Wird das Netzwerk aus allen Jugendlichen einer kompletten Klassenstufe (Baerveldt, Völker E Van Rossem 2008; Weerman 2011) oder sogar einer ganzen Stadt (Burk, Kerr E Stattin 2008) konstruiert, so kann ein positiver und signifikanter Effekt beobachtet werden.

Zwar stellen Schulfreunde eine wichtige Sozialisationsinstanz dar (z.B. Giordano 2003; Raufelder 2010), jedoch sind wir - in Übereinstimmung mit den oben genannten Ergebnissen - der Meinung, dass nicht außer Acht gelassen werden sollte, welchen Anteil die Schulfreunde an den Freunden insgesamt haben. Mit dem Fragebogen konnte dieser Anteil gemessen werden, und zur Erhöhung der Validität der vorliegenden Analysen reduzieren wir unsere Teilstichprobe weiter auf die Fälle, die ihre Freunde zum größten Teil ${ }^{3}$ in der Schulklasse haben. Diese weitere Reduktion führt zu einer Stichprobengröße von 1.045 Schülern. Bezogen auf die abhängige Variable Delinquenz unterscheiden sich diese Fälle vom Rest der Stichprobe (siehe unten Tabelle 1), was jedoch der Analyse von Zusammenhängen nicht im Wege steht.

\subsection{Abbängige und unabhängige Variablen}

Selbstberichtete Delinquenz: Die zu erklärende abhängige Variable ist die selbstberichtete Delinquenz der Jugendlichen. In der Schulbefragung wurde diese über eine aus 17 Items bestehende Skala gemessen, die strafbare und einige bedingt strafbare Handlungen erfasst (vgl. hierzu Oberwittler et al. 2014, 8 ff.). Im vorliegenden Fall wird über 13 der 17 Delikte hinweg die Inzidenz (Häufigkeit) aller in den letzten 12 Monaten begangenen strafbaren Handlungen ge-

3 Definition: nur Freunde (F) in der Schulklasse (SK) oder min. $1 \mathrm{~F}$ in SK und max. $1 \mathrm{~F}$ außerhalb SK oder min. $2 \mathrm{~F}$ in SK und max. $2 \mathrm{~F}$ außerhalb SK oder min. $3 \mathrm{~F}$ in SK und max. $3 \mathrm{~F}$ außerhalb SK oder mindestens $60 \%$ der F in SK. 
messen. Resultat ist eine Zählvariable, die entweder den Wert 0 oder eine positive natürliche Zahl als Wert annehmen kann, wobei der Maximalwert der Variable auf eine Obergrenze von 25 Delikten begrenzt wurde (siehe unten Tabelle 1). Die Inzidenzen der in die Zählvariable eingehenden Delikte wurden dabei vorab auf einen verteilungsabhängigen Maximalwert gedeckelt. Für die hier verwendete Skala haben wir das Item zum Drogenkonsum, das Item zum Verkauf von Raubkopien und die beiden Items zum Cyberbullying ausgeschlossen. Der Drogenkonsum würde mit sehr hohen Inzidenzen die Gesamtskala zu stark dominieren. Die Items zum Cyberbullying und zum Verkauf von Raubkopien weisen ebenfalls hohe Häufigkeiten auf. Bei diesen Items besteht zudem ein Validitätsproblem, da z.B. die Formulierung »jemanden im Internet beleidigt « einen weiten Interpretationsspielraum offen lässt. Beim Verkauf von Raubkopien hatte sich bereits während der Interviews gezeigt, dass dies z.T. mit dem bloßen Tauschen von Musikdateien oder dem Download von Musikdateien gleichgesetzt wurde.

Zur Erklärung der abhängigen Variable Delinquenz verwenden wir zwei aus der SAT abgeleitete Konzepte zur Messung delinquenter Neigungen und kriminogenen Gelegenheiten Jugendlicher sowie zwei unterschiedliche Maße zur Messung delinquenter Freunde. Alle Maße sind Konstrukte aus je mehreren Items, die als gemittelte Summenscores oder Faktorwerte in die Analysen eingehen.

Delinquente Neigung: Die Messung der delinquenten Neigungen erfolgt eng angelehnt an das bei Wikström et al. (2012) formulierte Konzept der »crime propensity «, was dort definiert ist als: »the tendency to see, and if so, to choose to break a rule of conduct (stated in law).«Die zwei Dimensionen dieses Konzepts sind moralisches Bewusstsein und Selbstkontrolle. Die Messung der Moralität besteht in unserer Untersuchung aus fünf Items zu devianten Überzeugungen, denen auf einer vierstufigen Skala zugestimmt oder widersprochen werden kann. Die fünf Items lauten: »Es ist ganz normal, jemanden zu schlagen, wenn man provoziert wird «, »Jeder Streit lässt sich auch durch Reden klären«, »Manche Konflikte lassen sich eben nur mit Gewalt lösen «, »Man sollte sich an die Gesetze halten, auch wenn die eigenen Interessen zu kurz kommen «, »Man kann ruhig mal was Verbotenes tun, wenn man sich dabei nicht erwischen lässt «.

Die Skala zur Messung niedriger Selbstkontrolle stellt eine reduzierte Version der von Grasmick et al. (1993) verwendeten Skala dar, die die Bereiche geringe Frustrationstoleranz (temper), risikoreiches Verhalten (risk seeking) und Hier- und Jetzt-Orientierung (impulsivity) anschneidet. Die Items lauten hierbei »Ich verliere ziemlich schnell die Beherrschung «, »Manchmal gehe ich Risiken ein nur so zum Spaß «, »Wenn ich echt wütend bin, dann sollten sich andere besser von mir fern halten «, »Spannung und Abenteuer sind mir wichtiger als Sicherheit « und »Ich handle oft spontan ohne lange nachzudenken«.

Beide Teilskalen wurden mit den Antwortoptionen »Stimmt genau «, »Stimmt eher«, »Stimmt kaum«, "Stimmt gar nicht « beantwortet und ergeben eine eindimensionale Skala (Cronbachs $\alpha=0.83$ ), welche die delinquente Neigung misst. Je höher der Wert der Skala, desto höher die delinquente Neigung.

Exposition/Gelegenheiten: In der PADS+-Studie konnte durch die innovative Messung der »space-time budgets « sehr genau ermittelt werden, wie viel Zeit Jugendliche in bestimmten Situationen verbringen (Wikström et al. 2012, 70 ff.). Zwar verfügen wir nicht über eine derartig genaue Messung, jedoch kann dieses Konzept auch mit unseren Daten ausreichend quantifiziert werden. Hierzu wurde ein Faktor ${ }^{4}$ aus sechs Items gebildet, der $54 \%$ der Va-

4 Die explorative Faktorenanalyse wurde auf Basis polychorischer Korrelationen durchgeführt. Gründe hierfür liegen in der ordinalen Datenstruktur und werden an anderen Stellen (z.B. Holgado-Tello et al. 2010) ausführlich diskutiert. Aufgrund der polychorischen Faktorenanalyse weicht der Mittelwert des Faktorscores von Null ab. 
rianz zwischen den Items erklären kann. Dieser kombinierte Index besteht zum einen aus vier Items, die explizit nach der Häufigkeit bestimmter Freizeitaktivitäten fragen. Die Frage »Wie oft machst du die folgenden Dinge in deiner Freizeit? « konnte dabei mit den Antwortoptionen »Nie«, »Manchmal«, »Oft« und »Sehr oft« beantwortet werden. Die vier Items zielen auf unstrukturierte Freizeitaktivitäten $\mathrm{ab}$ und lauten: »Sich mit Freunden/-innen draußen auf der Straße/einem Platz treffen und rumhängen «, »In die Disco/Clubs, zu Partys gehen «, »Abends mit anderen in einem Park/am See usw. abhängen und etwas trinken « und »In Kneipen gehen«. Zum anderen gehen zwei Fragen zur Häufigkeit und Dauer der mit Freunden verbrachten Zeit in den Index ein. Das erste Item fragt, wie oft sich die Jugendlichen nachmittags oder abends mit Freunden treffen. Die Antwortoptionen waren »Gar nicht «, »An 1 Tag«, »An 2 Tagen«, »An 3-4 Tagen« und »Öfter«. Das zweite Item fragt nach der Uhrzeit, zu der die Jugendlichen normalerweise unter der Woche nach Hause kommen. Die Antwortoptionen hierzu waren »Ich gehe abends während der Woche nicht weg «, »Bis $20 \mathrm{Uhr}$ «, »Bis $21 \mathrm{Uhr}$ «, »Bis $22 \mathrm{Uhr}$ «, »Bis $23 \mathrm{Uhr}$ «, »Bis $24 \mathrm{Uhr}$ « und »Nach $24 \mathrm{Uhr}$ «. Aus der Kombination dieser Variablen ergibt sich nun ein Maß dafür, bis zu welchem Grad die Jugendlichen ihre Freizeit in unstrukturierten und damit kriminogenen Situationen verbringen.

Delinquente Freunde: Wie bereits erwähnt, wird die Delinquenz der Freunde in unserer Untersuchung auf zwei unterschiedliche Arten gemessen. Die indirekte Messung erfolgt durch die in der Kriminologie konventionelle Methode, bei der die Befragten selbst Angaben zur Delinquenz ihrer Freunde machen (vgl. z.B. Elliott et al. 1983). Die Frage »Wie viele deiner Freunde/-innen haben schon mal folgende Dinge getan? « wurde dabei mit den Antwortoptionen »Keiner/keine«, »Einer/eine«, »Mehrere« und » Weiß nicht« beantwortet, wobei die Kategorie »Weiß nicht « der Kategorie »Keiner/keine« zugeordnet wurde. Bei dieser Messung wird also indirekt nach der Delinquenz der Freunde gefragt. Über die sechs Items »Etwas absichtlich beschädigt oder zerstört (z.B. in der Schule, in Parks, Telefonzellen, in der U-Bahn)«, »In einem Geschäft etwas gestohlen «, »Irgendwo eingebrochen, um etwas zu stehlen (in ein Haus, Keller, Laden usw.)«, »Jemandem mit Gewalt etwas weggenommen (durch Festhalten, Schlagen usw.) «, »Jemanden so geschlagen oder verprügelt, dass er/sie verletzt war oder blutete« und »Drogen genommen (Haschisch, Ecstasy usw.)« wurde eine eindimensionale Skala gebildet (Cronbachs $\alpha=0.75$ ), die die durchschnittliche Delinquenz der Freunde als Mittelwert wiedergibt. Der kleinstmögliche Wert ist dabei 0, der größtmögliche Wert ist 2. Die Delinquenz der Freunde der Schüler, die ihre Freunde hauptsächlich in der Klasse haben, liegt unterhalb $(\mathrm{M}=0.23$; siehe Tabelle 1) der Gruppe, die ihre Freunde eher außerhalb der Schulklasse haben $(\mathrm{M}=0.38)$.

Im Gegensatz hierzu erfolgt die direkte Messung durch die selbstberichtete Delinquenz der Freunde in der Schulklasse. Durch die Netzwerkdaten ist bekannt, mit welchen Schülern der Befragte befreundet ist. Somit können aus den Angaben der Freunde neue Variablen generiert werden, die verschiedene Eigenschaften der Freunde eines Befragten messen. Die sechs Items zur indirekten Messung der Delinquenz der Freunde finden sich wortgleich in der Skala zur selbstberichteten Delinquenz. Auf diese Weise ist es möglich, die indirekte Messung durch die gleiche Rechenoperation als direkte Messung zu replizieren. Für die Skala wurden nur die Prävalenzen der letzten zwölf Monate herangezogen, da sich die abhängige Variable ebenfalls nur auf die letzten zwölf Monate bezieht und die Peer-Delinquenz im Kontext der Gelegenheitsstrukturen betrachtet wird. Die Skala ist eindimensional und zufriedenstellend bezüglich ihrer internen Konsistenz (Cronbachs $\alpha=0.75$ ). Der durchschnittliche Wert ist für die Jugendlichen, die ihre Freunde hauptsächlich in der Klasse haben, etwas geringer als für die übrigen Schüler $(\mathrm{M}=0.32$ vs. $\mathrm{M}=0.37)$. 
Aus der Differenz der indirekten und direkten Messung lässt sich die Feblwabrnebmung berechnen, welche misst, ob Befragte die Delinquenz der Freunde eher unter- oder überschätzen. Streng genommen ist dieses Maß nur gültig für Schüler, die ihre Freunde ausschließlich in der Klasse haben.

\section{Tabelle $1 \quad$ Deskriptive Statistiken}

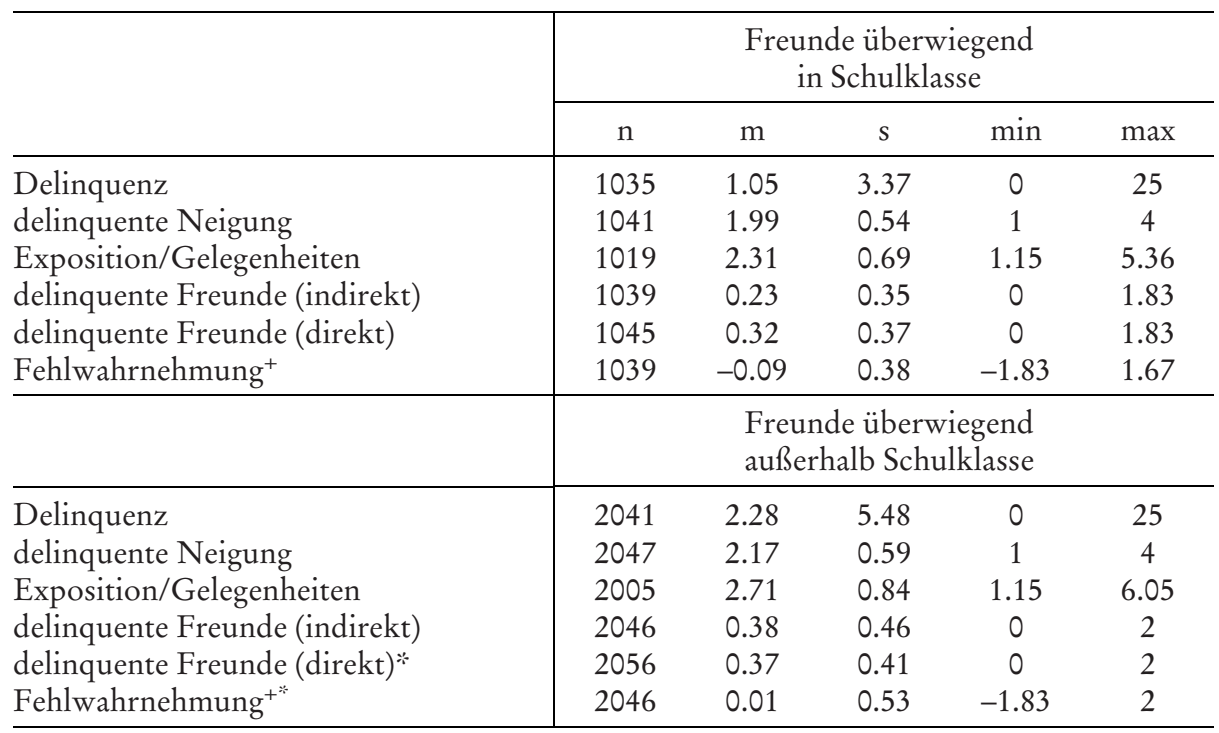

+ Fehlwahrnehmung = delinquente Freunde (indirekt) - delinquente Freunde (direkt) hohe Werte: Überschätzung / Werte um 0: balanciert / niedrige Werte: Unterschätzung

* Werte für Schüler mit überwiegend Freunden außerhalb der Schulklasse nur bedingt interpretierbar

\subsection{Analysestrategie}

Die Analysen werden nur für Befragte durchgeführt, deren Freunde hauptsächlich aus der Schulklasse sind, wobei alle unabhängigen Variablen für diese Teilstichprobe standardisiert (Z-Transformation) wurden. Durch den listenweisen Fallausschluss fehlender Werte verbleiben in den Analysen 1.007 gültige Fälle. In einem ersten Schritt werden die bivariaten $\mathrm{Zu}$ sammenhänge aller relevanten Variablen untersucht, was vor allem dazu dient, Unterschiede zwischen der direkten und indirekten Messung der delinquenten Freunde aufzuzeigen.

Anschließend werden die aus der SAT generierten Hypothesen in einem multivariaten Regressionsmodell getestet. Problematisch ist hierbei die schiefe Verteilung und die hohe Streuung der abhängigen Variablen Delinquenz, was dazu führt, dass die Parameterschätzung über ein negativ binomiales Modell erfolgen soll (z.B. Cameron E Trivedi 2010; Tutz 2010). Zwar gehört dieses zu der Familie der verallgemeinerten linearen Modelle, jedoch wird die Linearität in diesem Modell über eine logarithmische Linkfunktion hergestellt. Dies hat zur Folge, dass die Untersuchung von Interaktionen zwischen Variablen deutlich komplizierter wird. In der OLS-Regression werden Interaktionen in der Regel durch Aufnahme multiplikativer Terme getestet. Hierbei wird geprüft, ob der Effekt einer unabhängigen Variablen $x_{i}$ über unterschiedliche Ausprägungen der Variablen $x_{j}$ variiert (siehe Gleichung 1), die Effekte also nicht nur additiv, sondern auch multiplikativ wirken: 


$$
y=\beta_{0}+\beta_{1} x_{1}+\beta_{2} x_{2}+\beta_{3} x_{1} \times x_{2}+\varepsilon
$$

Betrachten wir nun Gleichung 2, so ist hier ebenfalls eine additive Strukturkomponente zu erkennen. Im Gegensatz zur OLS-Regression steht auf der linken Seite der Gleichung jedoch nicht ein der empirischen Verteilung entsprechender Schätzwert, sondern dessen natürlicher Logarithmus:

$$
\ln (y)=\beta_{0}+\beta_{1} x_{1}+\beta_{2} x_{2}+\varepsilon
$$

Durch Inversion der Gleichung kann dieser Wert zur Berechnung von Erwartungswerten der Skalierung der beobachteten Verteilung angepasst werden (Gleichung 3):

$$
\hat{y}=\exp \left(\beta_{0}\right) \times \exp \left(\beta_{1} x_{1}\right) \times \exp \left(\beta_{2} x_{2}\right)
$$

Dies hat allerdings zur Folge, dass die Strukturkomponente nunmehr multiplikativ ist. Dementsprechend haben nichtlineare Modelle stets modellinhärente Interaktionen, wodurch die Analyse von Interaktionen in derartigen Modellen generell problematisch wird (für eine ausführliche Darstellung siehe Oberwittler E Gerstner 2014). Da modellinhärente Interaktionen zwischen allen Kovariaten stets implizite Begleiterscheinung nichtlinearer Regressionsmodelle sind, wird in diesem Zusammenhang auch oft von konditionalen Effekten gesprochen (Tsai E Gill 2013). Den Empfehlungen Greenes (2010) folgend wählen wir eine praktikable Lösung zur Untersuchung der Interaktionseffekte zum einen durch die graphische Darstellung der modellierten Zusammenhänge und zum anderen durch die Analyse marginaler Effekte. Die hierbei berechneten marginalen Effekte repräsentieren als partielle Ableitung $(d y / d x)$ eine lokale Steigung der aus dem negativen Binomialmodell resultierenden Funktion für theoretisch relevante Wertekombinationen der Prädiktoren. Somit kann beispielsweise untersucht werden, ob der Effekt der Exposition konstant ist, wenn Jugendliche eine geringe oder hohe individuelle delinquente Neigung aufweisen. Den Annahmen der SAT entsprechend müsste dieser Effekt variieren. ${ }^{5}$ Ergänzend zu diesen marginalen Effekten an repräsentativen Werten (MER = marginal effect at a representative value) bietet das Programm Stata (StataCorp. 2013) die Option, marginale Effekte als Durchschnittswert aller Beobachtungen zu berechnen (AME = average marginal effect). Dies eignet sich besonders zur Beurteilung der Effektstärken bzw. der Wichtigkeit einzelner Prädiktoren, da die AMEs den unstandardisierten Koeffizienten eines OLS-Modells mit den gleichen Daten in der Regel recht nahe kommen (Cameron E Trivedi 2010, 350; Mood 2010). Beachtet werden muss hierbei, dass dies der durchschnittliche Effekt für alle Befragten ist, nicht jedoch der Effekt für den durchschnittlichen Befragten. Für die Beurteilung der Interaktion bietet dieser Durchschnittseffekt nur bedingt zusätzliche Information (s.u.).

Es sei an dieser Stelle auch erwähnt, dass in den Modellen auf Kontrollvariablen verzichtet wird. Diese haben keine theoretisch bedeutsame Rolle und würden die Komplexität der Modelle zusätzlich erhöhen und unsere Art der Interpretation der Interaktionen erschweren. ${ }^{6}$

5 Repräsentative Werte werden von uns im Folgenden vorwiegend über Quantile definiert. Das 5-\%-Quantil ( $Q_{05}$ ) steht dabei für geringe/niedrige Ausprägungen. Mittelhohe Werte einer Variablen werden über das 75-\%-Quantil $\left(\mathrm{Q}_{75}\right)$ und hohe Ausprägungen über das 95-\%-Quantil $\left(\mathrm{Q}_{95}\right)$ definiert. Bei den delinquenten Freunden in Abbildung 3 und 4 steht »niedrig « für das 5-\%-Quantil, »mittel « für das arithmetische Mittel, »hoch « für das arithmetische Mittel + eine Standardabweichung und »sehr hoch « für das 95-\%-Quantil.

6 Alle Modelle und darauf basierende Grafiken wurden alternativ auch unter Kontrolle der Variablen Geschlecht berechnet. Die Ergebnisse und Bewertungen unserer Hypothesen ändern sich hierdurch nicht. Die Ergebnisse können auf Anfrage über die Autoren bezogen werden. 
Alle Modelle wurden mit robusten Standardfehlern für die in Schulklassen geklumpten Analyseeinheiten geschätzt. ${ }^{7}$

\section{Ergebnisse}

Tabelle 2 zeigt eine Korrelationsmatrix der relevanten Variablen. Bezüglich der bivariaten Zusammenhänge mit der eigenen Delinquenz der Jugendlichen ist zu beobachten, dass alle Variablen wie erwartet einen positiven Zusammenhang aufweisen. Für uns ist der Zusammenhang zwischen der eigenen Delinquenz und der Delinquenz der Freunde von besonderem Interesse. Diese Korrelation ist für die konventionelle, indirekte Messung deutlich stärker $\left(r_{s}=0.48 ; p<0.001\right)$ als für die direkte Messung über Netzwerkdaten $\left(r_{s}=0.28\right.$; $p<0.001$ ). Ein ähnliches Ergebnis wird auch bei Weerman E Smeenk (2005) sowie bei Meldrum et al. (2009) berichtet. Die Unterschiede lassen sich darauf zurückführen, dass die indirekte Messung durch die Projektion der eigenen Delinquenz auf die Delinquenz der Freunde verzerrt ist, wodurch der Zusammenhang überschätzt wird. Betrachtet man die Korrelation zwischen der Fehlwahrnehmung der Peer-Delinquenz und der eigenen Delinquenz $\left(r_{s}=0.16 ; p<0.001\right)$, so zeigt sich, dass ebenfalls ein positiver Zusammenhang besteht. Je höher die eigene Delinquenz, desto eher wird die Delinquenz der Freunde überschätzt; dieser Zusammenhang wird auch bei Young et al. (2011) beobachtet. Außerdem berichten die Autoren einen negativen Zusammenhang der Fehlwahrnehmung mit dem Konzept Selbstkontrolle. Analog hierzu ist in unseren Daten ein positiver Zusammenhang mit der delinquenten Neigung $\left(r_{s}=0.15 ; p<0.001\right)$ zu beobachten. Welche Konsequenzen haben diese Beobachtungen nun bezüglich der Bewertung delinquenter Peers innerhalb der SAT?

Tabelle 2 Bivariate Korrelationen/Rangkorrelation nach Spearman

\begin{tabular}{|c|c|c|c|c|c|c|}
\hline Variablen & (1) & (2) & (3) & (4) & $(5)$ & (6) \\
\hline (1) Delinquenz & - & & & & & \\
\hline (2) delinquente Neigung & $0.43 * * *$ & - & & & & \\
\hline (3) Exposition/Gelegenheiten & $0.29 \% * \%$ & $0.38 \% * \%$ & - & & & \\
\hline $\begin{array}{l}\text { (4) delinquente Freunde } \\
\text { (indirekt) }\end{array}$ & $0.48 * * *$ & $0.47 \% * \%$ & $0.39 * * *$ & - & & \\
\hline $\begin{array}{l}\text { (5) delinquente Freunde } \\
\text { (direkt) }\end{array}$ & $0.28 * *$ & $0.25 \% *$ & $0.26 * * *$ & $0.41 * *$ & - & \\
\hline (6) Fehlwahrnehmung ${ }^{+}$ & $0.16 * * *$ & $0.15 * * \%$ & $0.09 * \%$ & $0.42 * * *$ & $-0.58 \% * \%$ & - \\
\hline
\end{tabular}

$* \mathrm{p}<0.05 * \mathrm{p}<0.01 * * \mathrm{p}<0.001$

$\mathrm{n}=1.007$

+ Fehlwahrnehmung $=$ delinquente Freunde (indirekt) - delinquente Freunde (direkt) hohe Werte: Überschätzung / Werte um 0: balanciert / niedrige Werte: Unterschätzung

Die folgenden Regressionsmodelle (Tabelle 3) untersuchen die multivariaten Zusammenhänge zwischen der abhängigen Variablen Delinquenz und den Kovariaten kriminelle Neigung, Exposition/Gelegenheiten und delinquente Freunde. Dabei werden die modellinhärenten Interaktionen zwischen den Kovariaten im Nachgang der Schätzung untersucht. Dies geschieht durch grafische Darstellung vorhergesagter Werte und der Analyse marginaler Effekte. Modell 1 und Modell 2 beinhalten jeweils nur den Effekt der kriminellen Neigung oder

7 Stata option vce (cluster clustvar), mit clustvar = Klassen-ID. 
der Exposition, die beide statistisch signifikant sind und deren positives Vorzeichen bedeutet, dass höhere Werte mit mehr Delinquenz einhergehen. Die Zusammenhänge bleiben auch dann bestehen, wenn beide Effekte simultan geschätzt werden. Dieses Ergebnis steht in Einklang mit den Hypothesen $\mathrm{H} 1$ und H2, die jeweils einen positiven Zusammenhang der beiden Prädiktoren mit der selbstberichteten Delinquenz supponieren.

Sollen nun die Annahmen der SAT überprüft werden, so kann untersucht werden, ob die beiden Prädiktoren in einem interaktiven Verhältnis stehen, d.h. in welcher Form der Effekt des einen Prädiktors auf die Delinquenz an die Ausprägung des anderen Prädiktors gekoppelt ist. Hypothese 3 kann beispielsweise dahingehend untersucht werden, ob vorhandene Tatgelegenheiten (Exposition) immer mit höherer Kriminalität einhergehen oder ob diese entsprechend dem PCP nur dann eine Rolle spielen, wenn auch eine delinquente Neigung vorhanden ist. Umgekehrt kann auch danach gefragt werden, ob eine hohe delinquente Neigung immer den gleichen Effekt hat, oder ob dieser davon abhängt, welchen Situationen man ausgesetzt ist. Dieses Zusammenspiel kann anhand der Abbildung 1 untersucht werden. Auf der y-Achse sind die aus Modell 3 vorhergesagten Werte für die eigene Delinquenz abgetragen. Die x-Achse misst die Exposition bzw. die Gelegenheitsstrukturen. Die durchgezogene Linie stellt den Zusammenhang für die Jugendlichen mit geringer krimineller Neigung dar. Hier ist zu sehen, dass die Delinquenz mit Zunahme der Gelegenheiten nicht zunimmt. Ferner gilt, dass diese Gruppe eigentlich überhaupt nicht delinquent ist. Bei der Gruppe mit mittelhoher delinquenter Neigung ist bei geringer Exposition ebenfalls nur sehr wenig Delinquenz zu erwarten (gestrichelte Linie). Mit steigenden Gelegenheiten ist jedoch eine deutliche Zunahme der vorhergesagten Werte zu beobachten. Deutlich ausgeprägter ist diese Steigung, wenn die delinquente Neigung hoch ist (gepunktete Linie). Zwar sind für Jugendliche mit hoher delinquenter Neigung generell mehr Straftaten zu erwarten, besonders hohe Werte auf der Delinquenz-Skala kommen jedoch dann zustande, wenn die kriminelle Neigung hoch ist und durch häufige unstrukturierte Freizeitaktivitäten mit Freunden viele Gelegenheiten zum Begehen von Straftaten vorhanden sind. Dieses Ergebnis bestätigt die von uns formulierte Hypothese 3 und deckt sich mit Ergebnissen, die bei Wikström et al. (2012) berichtet werden. Im Folgenden soll nun der Frage nachgegangen werden, wie dieses Zusammenspiel aussieht, wenn der Effekt delinquenter Freunde in die Gleichung aufgenommen wird.

Tabelle 3 Negative Binomialregression, abhängige Variable Delinquenz

\begin{tabular}{l|ccccc}
\hline Delinquenz & $(1)$ & $(2)$ & $(3)$ & $(4)$ & $(5)$ \\
\hline delinquente Neigung & $1.314^{* * *}$ & - & $1.062^{* * * *}$ & $0.842^{* * * *}$ & $1.020^{* * * *}$ \\
& $(11.18)$ & & $(10.26)$ & $(7.52)$ & $(9.50)$ \\
Exposition/Gelegenheiten & - & $0.799^{* * *}$ & $0.463^{* * *}$ & 0.107 & $0.436^{* * *}$ \\
& & $(7.11)$ & $(3.28)$ & $(0.63)$ & $(2.93)$ \\
delinquente Freunde (ind.) & - & - & - & $0.757^{* * * *}$ & - \\
delinquente Freunde (dir.) & - & - & - & $(6.95)$ & \\
Konstante & & & - & $0.291^{* * *}$ \\
& $-0.661^{* * *}$ & $-0.342^{* *}$ & $-0.747^{* * *}$ & $-0.932^{* * *}$ & $-0.753^{* * *}$ \\
$\mathrm{n}$ & $(-4.78)$ & $(-2.67)$ & $(-4.83)$ & $(-6.65)$ & $(-4.50)$ \\
\hline
\end{tabular}

robuste Standardfehler geclustert über Schulklassen

z-Statistik in Klammern

$* \mathrm{p}<0.05 * \mathrm{p}<0.01 * * * \mathrm{p}<0.001$ 
Abbildung 1 2-Wege-Interaktion

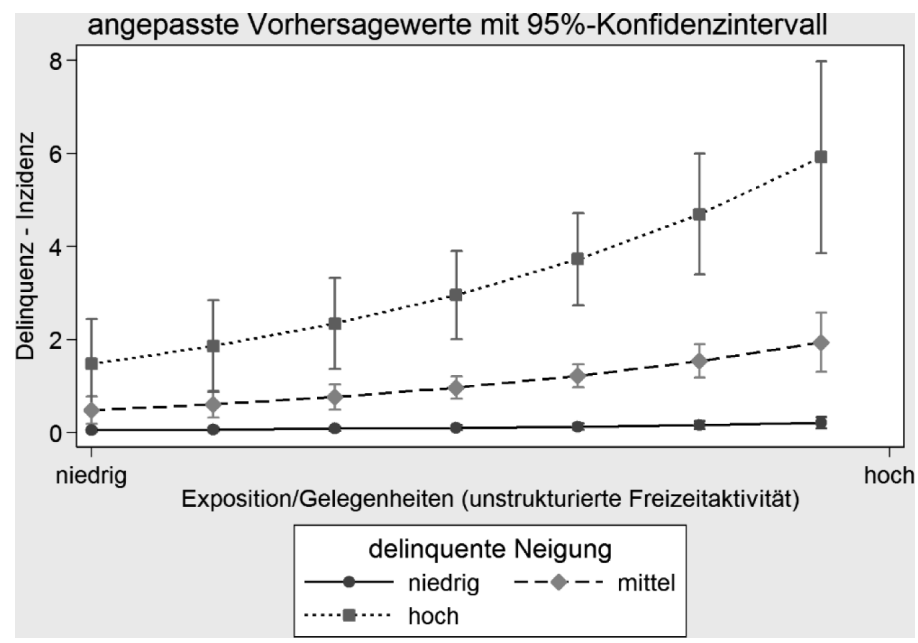

Zusammenhänge zwischen eigener Delinquenz und delinquenten Freunden können in ihren Wirkmechanismen sehr unterschiedlich untersucht werden. Eine Begrenzung ergibt sich durch Querschnittsdaten, welche es z.B. nur schwer zulassen, Zusammenhänge unter lerntheoretischen Aspekten zu interpretieren, da diese auf Veränderungsprozesse ausgerichtet sind. Fragestellungen, die die Wirkungsmechanismen delinquenter Freunde eher den Gelegenheitsstrukturen zuordnen, sind jedoch mit Querschnittsdaten möglich. Svensson $\mathcal{E}$ Oberwittler (2010) können z.B. für Daten aus Schweden und Deutschland nachweisen, dass der Effekt unstrukturierter Freizeitaktivitäten mit der Delinquenz der Freunde interagiert, wobei kein delinquenzfördernder Effekt besteht, wenn die Freunde nicht oder nur wenig delinquent sind, dieser Effekt jedoch bei vielen delinquenten Freunden besonders ausgeprägt ist. Ausgehend von dieser Beobachtung soll nun getestet werden, wie sich die Interaktion aus Modell 3 ändert, wenn eine Messung der delinquenten Freunde in das Modell aufgenommen wird. Modell 4 (Tabelle 3) beinhaltet dabei die indirekte Messung, die auch von Svensson E Oberwittler (2010) verwendet wurde. Ob dabei der Einfluss der indirekten Messung delinquenter Freunde überschätzt wird, wie z.B. bei Haynie (2001) oder Haynie E Osgood (2005) dargelegt, wird durch den Vergleich mit Modell 5 (Tabelle 3) geprüft, das alternativ die direkte Messung der delinquenten Freunde nutzt.

In Modell 4 wird deutlich, dass sowohl die individuelle delinquente Neigung als auch die delinquenten Freunde einen signifikanten Einfluss auf die Delinquenz haben. Im Gegensatz dazu ist der Effekt für die Exposition nicht signifikant. Dies würde den Schluss nahelegen, dass für die Vorhersage der Delinquenz nicht relevant ist, was man macht, sondern lediglich, welche individuellen Neigungen man mitbringt und welche Freunde man hat. Verwendet man jedoch alternativ die direkte Messung der delinquenten Freunde (Modell 5), so weisen alle drei Prädiktoren einen signifikanten und positiven Effekt auf, auch die Gelegenheiten. Wie schon erwähnt, folgt die Interpretation der Regressionskoeffizienten in nichtlinearen Regressionsmodellen anderen Regeln als in der linearen OLS-Regression. Im OLS-Modell ohne Interaktionsterme sind die Steigerungskoeffizienten für alle Einheiten bzw. Befragten identisch. Im nichtlinearen Modell betreffen die im Modell berichteten Koeffizienten jedoch 
nur den »durchschnittlichen« Befragten. Mit jeglicher Variation der Ausprägungen der Prädiktoren ändert sich dabei der Effekt eines bestimmten Prädiktors, was bereits in Bezug auf die modellinhärenten Interaktionen ausgeführt wurde. Um dieses Problem zu umgehen, können für alle Prädiktoren die durchschnittlichen marginalen Effekte (average marginal effects, AME) berechnet werden, die der Interpretation der OLS-Koeffizienten nahe kommen. Mit der Verschiebung des Wertes $x_{i}$ um eine Einheit ändert sich der Wert der vorhergesagten Variablen $\hat{y}$ durchschnittlich um den Wert des Koeffizienten. Wir verzichten in diesem Zusammenhang auf die ausführliche Interpretation von Tabellen und betrachten die AMEs grafisch. Abbildung 2 zeigt die AMEs mit einem 95-\%-Konfidenzintervall für die Modelle 4 und 5. Dabei ergibt sich ein ähnliches Bild wie durch die Modellkoeffizienten: Die indirekte Messung der delinquenten Freunde, die in Teilen die eigene Delinquenz reflektiert, hat einen ähnlich starken Effekt wie die delinquente Neigung, und der AME der Gelegenheiten ist nicht signifikant (linke Teilgrafik). Zieht man zur Analyse alternativ die direkte Messung heran, so sind die AMEs aller drei Prädiktoren signifikant. Es kann an dieser Stelle festgehalten werden, dass die Hypothese 4 - delinquente Freunde gehen mit höherer eigener Delinquenz einher - für beide Messungen delinquenter Freunde ihrer Nullhypothese standhält. Die indirekte Messung überlagert jedoch den Effekt der Gelegenheiten, wobei diese Beobachtung mit Hypothese 6 in Einklang steht.

\section{Abbildung 2 Durchschnittliche marginale Effekte (AMEs) Modell 4 und 5}

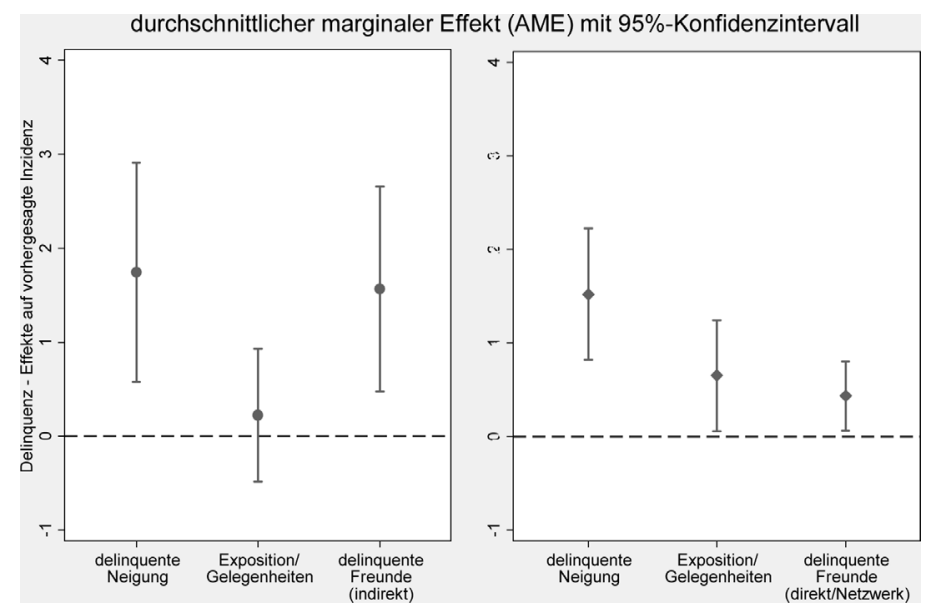

Welche Implikationen diese Ergebnisse empirisch auf die Interaktion zwischen individueller Neigung und der Exposition sowie theoretisch auf den »perception-choice process « haben, lässt sich wie für Modell 3 grafisch untersuchen. Aufgrund der konditionalen Abhängigkeit der Koeffizienten gilt für die Modelle 4 und 5, dass für die Untersuchung der Hypothese 5 von einer 3-Wege-Interaktion ausgegangen werden muss. Abbildung 3 bezieht sich auf das Modell 4. Hier ist zu erkennen, dass mit mehr delinquenten Freunden eine höhere eigene Delinquenz einhergeht, und diese Zunahme auch mit der individuellen Neigung in Zusammenhang steht. Mit Zunahme der kriminogenen Gelegenheiten ist jedoch in keiner der vier Teilgrafiken eine bedeutsame Zunahme der Delinquenz zu beobachten. Die Linien für die unterschiedlichen Ausprägungen der delinquenten Neigung verlaufen hierbei immer nahezu 
parallel, was in Einklang mit dem nicht-signifikanten durchschnittlichen marginalen Effekt für diesen Prädiktor steht. Im Vergleich hierzu kann man in Abbildung 4 für alle vier Teilgrafiken die Interaktion zwischen delinquenter Neigung und den Gelegenheiten erkennen. Die Linien für die Ausprägungen der delinquenten Neigung verlaufen nicht parallel, sondern gehen mit Zunahme der Gelegenheiten in der für Interaktionen typischen Fächerform auseinander. Die Interaktion wird mit Zunahme des Werts für die delinquenten Freunde ausgeprägter, was auf eine 3-Wege-Interaktion hindeutet.

Abbildung 3 3-Wege-Interaktion, indirekte Messung delinquenter Peers (Angaben Befragter)

angepasste Vorhersagewerte mit 95\%-Konfidenzintervall

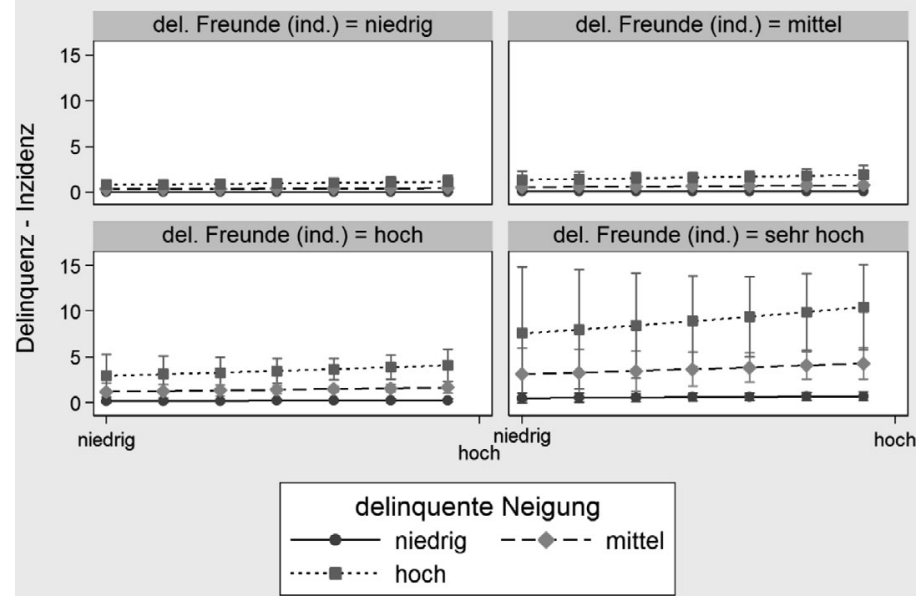

Abbildung 4 3-Wege-Interaktion, direkte Messung delinquenter Peers (Netzwerk)

angepasste Vorhersagewerte mit 95\%-Konfidenzintervall

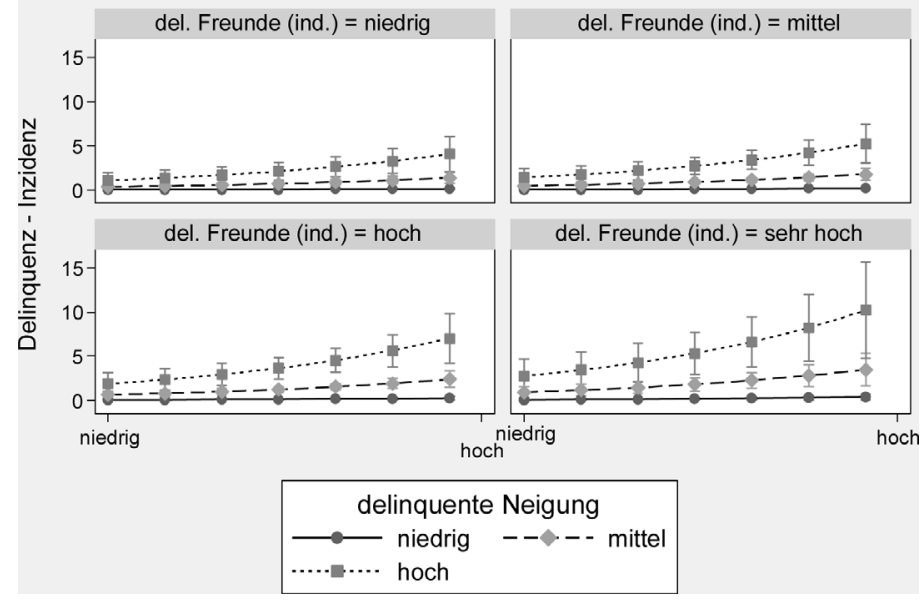


Die Frage nach den Interaktionen lässt sich auch über marginale Effekte an repräsentativen Werten (MERs) betrachten. In Tabelle 4 wird der durchschnittliche marginale Effekt für die Exposition für unterschiedliche Ausprägungen individueller Neigung und delinquenter Freunde berichtet. Der obere Teil der Tabelle (Zeile 1-15) bezieht sich dabei auf das Modell 4

Tabelle 4 Marginale Effekte an repräsentativen Werten (MERs), Modell 4 und 5

\begin{tabular}{|c|c|c|c|c|c|c|c|c|c|c|c|c|}
\hline & Zeile & $A$ & $B$ & C & $D$ & & $E$ & $F$ & $G$ & $H$ & $I$ & $J$ \\
\hline & & DF & $\mathbf{P}$ & $\begin{array}{c}\text { E MER }_{i} \\
d y / d x\end{array}$ & $\mathrm{P}>|z|$ & & $\mathrm{DF}$ & P & $\begin{array}{c}\text { E MER }_{j} \\
\text { dy/dx }\end{array}$ & $\mathrm{P}>|z|$ & $\begin{array}{c}\text { Kontrast } \\
\mathrm{dy} / \mathrm{dx}\end{array}$ & $\mathrm{P}>|z|$ \\
\hline \multirow{15}{*}{$\begin{array}{l}\text { delin- } \\
\text { quente } \\
\text { Freunde: } \\
\text { indirekte } \\
\text { Messung } \\
\text { (Modell 4) }\end{array}$} & 1 & ○ & ○ & .009 & 0.458 & vs. & o & 0 & .055 & 0.444 & 0.045 & 0.443 \\
\hline & 2 & $\circ$ & ○ & .009 & 0.458 & vs. & ○ & $\bullet$ & .133 & 0.442 & 0.123 & 0.442 \\
\hline & 3 & ○ & o & .009 & 0.458 & vs. & • & o & .093 & 0.394 & 0.084 & 0.389 \\
\hline & 4 & ० & o & .009 & 0.458 & vs. & • & 0 & .548 & 0.373 & 0.538 & 0.371 \\
\hline & 5 & 0 & ○ & .009 & 0.458 & vs. & • & $\bullet$ & 1.327 & 0.369 & 1.317 & 0.369 \\
\hline & 6 & o & 0 & .055 & 0.444 & vs. & o & • & .133 & 0.442 & 0.078 & 0.443 \\
\hline & 7 & ० & 0 & .055 & 0.444 & vs. & • & $\circ$ & .093 & 0.394 & 0.039 & 0.424 \\
\hline & 8 & o & 0 & .055 & 0.444 & vs. & • & 0 & .548 & 0.373 & 0.493 & 0.366 \\
\hline & 9 & o & 0 & .055 & 0.444 & vs. & • & $\bullet$ & 1.327 & 0.369 & 1.272 & 0.366 \\
\hline & 10 & o & $\bullet$ & .133 & 0.442 & vs. & • & o & .093 & 0.394 & -0.039 & 0.640 \\
\hline & 11 & o & • & .133 & 0.442 & vs. & • & 0 & .548 & 0.373 & 0.415 & 0.357 \\
\hline & 12 & 0 & • & .133 & 0.442 & vs. & • & - & 1.327 & 0.369 & 1.194 & 0.362 \\
\hline & 13 & • & ○ & .093 & 0.394 & vs. & • & 0 & .548 & 0.373 & 0.454 & 0.370 \\
\hline & 14 & • & $\circ$ & .093 & 0.394 & vs. & • & - & 1.327 & 0.369 & 1.233 & 0.369 \\
\hline & 15 & $\bullet$ & 0 & .548 & 0.373 & vs. & • & • & 1.327 & 0.369 & 0.779 & 0.370 \\
\hline \multirow{15}{*}{$\begin{array}{l}\text { delin- } \\
\text { quente } \\
\text { Freunde: } \\
\text { direkte } \\
\text { Messung } \\
\text { (Modell 5) }\end{array}$} & 16 & 0 & o & .039 & 0.025 & vs. & 0 & 0 & .337 & 0.006 & 0.298 & 0.006 \\
\hline & 17 & o & o & .039 & 0.025 & vs. & ○ & - & .986 & 0.007 & 0.947 & 0.008 \\
\hline & 18 & o & ○ & .039 & 0.025 & vs. & • & $\circ$ & .098 & 0.044 & 0.059 & 0.112 \\
\hline & 19 & 0 & ○ & .039 & 0.025 & vs. & • & 0 & 0.843 & 0.014 & 0.803 & 0.016 \\
\hline & 20 & o & $\circ$ & .039 & 0.025 & vs. & • & - & 2.465 & 0.014 & 2.425 & 0.014 \\
\hline & 21 & ○ & 0 & .337 & 0.006 & vs. & o & • & .986 & 0.007 & 0.649 & 0.010 \\
\hline & 22 & o & 0 & .337 & 0.006 & vs. & • & ○ & .098 & 0.044 & -0.239 & 0.020 \\
\hline & 23 & o & & .337 & 0.006 & vs. & • & 0 & 0.843 & 0.014 & 0.505 & 0.070 \\
\hline & 24 & o & 0 & .337 & 0.006 & vs. & • & - & 2.465 & 0.014 & 2.128 & 0.022 \\
\hline & 25 & o & $\bullet$ & .986 & 0.007 & vs. & • & $\circ$ & .098 & 0.044 & -0.888 & 0.011 \\
\hline & 26 & 0 & • & .986 & 0.007 & vs. & • & 0 & 0.843 & 0.014 & -0.144 & 0.655 \\
\hline & 27 & o & • & .986 & 0.007 & vs. & • & - & 2.465 & 0.014 & 1.479 & 0.067 \\
\hline & 28 & • & ○ & .098 & 0.044 & vs. & • & 0 & 0.843 & 0.014 & 0.744 & 0.013 \\
\hline & 29 & • & 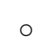 & .098 & 0.044 & vs. & • & - & 2.465 & 0.014 & 2.366 & 0.014 \\
\hline & 30 & • & 0 & .843 & 0.014 & vs. & • & • & 2.465 & 0.014 & 1.622 & 0.016 \\
\hline
\end{tabular}

$\mathrm{DF}=$ delinquente Freunde

$\mathrm{P}=$ kriminelle Neigung (crime propensity)

$\mathrm{E}=$ Exposition/Gelegenheiten (criminogenic exposure)

MER dy/dx = durchschnittlicher marginaler Effekt von Exposition/Gelegenheiten an repräsentativen Werten für $\mathrm{P}$ und $\mathrm{DF}$ (dy/dx = partielle Ableitung)

Kontrast dy/dx = E MERj dy/dx - E MERi dy/dx

$\circ=$ niedrig (Q05), $\bigcirc=$ mittelhoch $(\mathrm{Q} 75), \bullet=$ hoch $(\mathrm{Q} 95)$ 
mit indirekt gemessener Peer-Delinquenz. Der untere Tabellenteil bezieht sich auf Modell 5 und damit auf die direkt gemessene Peer-Delinquenz. Pro Modell wurden sechs Kombinationen für niedrige und hohe Werte der Peer-Delinquenz sowie niedrige, mittelhohe und hohe Werte für die individuelle Neigung berechnet.

Jede Kombination gibt an, ob der durchschnittliche marginale Effekt der Exposition an dieser Stelle signifikant ist. Für Modell 4 trifft das auf keine der Kombinationen zu, was letztlich dazu führt, dass auch die Kontraste bzw. die Differenzen zwischen den berechneten MERs sich statistisch nicht von Null unterscheiden. Im Gegensatz dazu sind alle sechs berechneten MERs für Modell 5 statistisch signifikant. Für die Kombination niedrige individuelle Neigung und wenig delinquente Freunde (Zeile 16, Spalte C [16/C]) ergibt sich ein durchschnittlicher Effekt von 0.039. Mit Verschiebung der Exposition um eine Einheit (hier: $\mathrm{sd}=1$ ) an dieser Kombination ändert sich die Delinquenz um durchschnittlich 0.039 Taten, was ein sehr geringer Wert ist. Geht man von einer Kombination mit mittelhoher delinquenter Neigung und ebenfalls wenig delinquenten Freunden [16/G] aus, so ändert sich die Delinquenz um durchschnittlich 0.337. Der Effekt der Exposition ist an dieser Stelle also im Mittel um das Zehnfache erhöht. Vergleicht man beide Kombinationen, ergibt sich ein Kontrastwert, der aus der Differenz der beiden durchschnittlichen marginalen Effekte besteht. Für diese Differenz $([16 / \mathrm{G}]-[16 / \mathrm{C}]=0.337-0.039=0.298)$ lässt sich eine Irrtumswahrscheinlichkeit $(\mathrm{P}>|z|=0.006)$ berechnen, über die geprüft werden kann, ob der Unterschied signifikant von Null verschieden ist und damit der durchschnittliche marginale Effekt der Exposition für diese Kombination variiert. Das Zahlenbeispiel deutet auf eine Interaktion hin. Ebenso ist der uns interessierende Effekt für die Kombination von vielen delinquenten Freunden und hoher individueller Neigung [30/G] signifikant verschieden [30/J] und im Mittel um das Dreifache höher als für die Kombination von vielen delinquenten Freunden und mittelhoher individueller Neigung. Durch die Kombinationen aus Tabelle 4 können noch weitere solcher Unterschiede festgestellt oder alternativ auch aus Kombinationen von delinquenter Neigung und Exposition geprüft werden, wie der Effekt der delinquenten Freunde variiert, oder es kann auf ähnliche Weise untersucht werden, wie Effekte bei Kombinationen repräsentativer Werte aller drei Prädiktoren (vgl. hierzu Abbildungen 3 und 4) variieren.

Es wird an dieser Stelle klar, dass die Modellierung und Interpretation von Interaktionen in nichtlinearen Modellen schwierig ist und - nicht nur unserer Meinung nach - weiterer Bedarf an Forschung und Innovation wünschenswert ist (z.B. Ai E Norton 2003; Greene 2010; Karaca-Mandic, Norton E Dowd 2012; Tsai E Gill 2013). Zu der Beurteilung von Hypothese 5 lässt sich jedoch das Folgende sagen: Je nach Messung der delinquenten Freunde ist diese zu verwerfen oder beizubehalten. Mit der indirekten Messung (Modell 4) muss die Hypothese verworfen werden. Die Interaktion variiert nicht mit der Ausprägung delinquenter Freunde, und zwar deshalb, weil die Variable Exposition/Gelegenheiten in dem Modell keinerlei Relevanz hat. Für die eigene Delinquenz der Jugendlichen ist es nicht relevant, was sie machen, sondern eine höhere Delinquenz ergibt sich nur durch die eigene delinquente Neigung sowie die Delinquenz der Freunde. Die Gelegenheiten spielen im Rahmen des "perception-choice process « keine Rolle.

Bei Verwendung der direkten Messung der Peer-Delinquenz dagegen hält Hypothese 5 der empirischen Überprüfung stand. Für das Ergebnis des "perception choice process « kommt es darauf an, welche individuellen kriminellen Neigungen man mitbringt, was man in seiner Freizeit mit seinen Freunden macht und wer diese Freunde sind. Beispielsweise wird das Zusammenspiel aus individueller Neigung und der Exposition umso ausgeprägter, je delinquenter die Freunde sind. Aus den Modellvergleichen wird deutlich, dass die Art der Messung delinquenter Freunde Einfluss auf die empirische Überprüfung von Hypothesen 
nehmen kann. Hypothese 6 soll hier als bestätigt angesehen werden. Weitere Überlegungen, die sich hieraus ergeben, sowie die zusammenfassende Bewertung unserer Analysen soll im Folgenden diskutiert werden.

\section{Diskussion}

Mit zahlreichen Studien aus unterschiedlichen Ländern konnten die Annahmen der Situational Action Theory empirisch überprüft und bestätigt werden. Auch mit den Daten der "Schulbefragung Lebenslagen und Risiken von Jugendlichen«, die in zwei deutschen Großstädten erhoben wurden, konnten die zentralen Annahmen der SAT bestätigt werden. Es konnte gezeigt werden, dass unstrukturierte Freizeitaktivitäten und persönliche kriminelle Neigungen einen delinquenzfördernden Effekt haben. Jugendliche mit risikoreichem Freizeitstil werden jedoch nicht zwangsläufig delinquent, sondern dies hängt von deren individueller Neigung zu kriminellem Verhalten ab, welche den Effekt der kriminogenen Situationen moderiert. In Bezug auf den "perception-choice process « wirkt die individuelle kriminelle Neigung als moralischer Filter (Wikström et al. 2012, 24), der für abweichendes Verhalten in einer bestimmten Situation durchlässig oder nicht durchlässig ist. Die Situation ist vor allem daran gebunden, was die Jugendlichen häufig machen. Unstrukturierte Freizeitaktivitäten mit Freunden ohne Beaufsichtigung durch Erwachsene wie das »Rumhängen « auf Straßen, Plätzen oder in Parks können als kriminogen gelten, umso mehr, wenn Jugendliche dies während der Woche abends länger tun.

In unseren Analysen sind wir aber auch der Frage nachgegangen, welche Rolle die Delinquenz der Freunde spielt, oder in anderen Worten: mit wem die Jugendlichen ihre Freizeit verbringen. In bisherigen Untersuchungen zur SATwurde dieser Aspekt häufig der Messung der kriminogenen Situation zugerechnet (z.B. Svensson E Paurwels 2010; Wikström E Svensson 2008), wobei u.a. davon ausgegangen wird, dass die Delinquenz der Freunde ein Indikator für die kriminellen Neigungen der Freunde darstellt (Wikström et al. 2012, 152). In unseren Analysen wurde die Peer-Delinquenz als eigenständiger Effekt neben den unstrukturierten Freizeitaktivitäten betrachtet. Unter Verwendung von zwei unterschiedlichen Messungen der Peer-Delinquenz kommen wir zu wichtigen methodischen und theoretischen Schlüssen, die nicht unabhängig voneinander betrachtet werden können. Aus der aktuellen Forschung zur Messung der Peer-Delinquenz ist bekannt, dass diese verzerrt ist, wenn die Angaben hierüber von den Befragten selbst stammen (indirekte Messung), wobei die eigene Delinquenz häufig auf die Freunde projiziert wird. Eine Konsequenz hieraus ist, dass der Zusammenhang zwischen eigener Delinquenz und der Delinquenz der Freunde überschätzt wird. Dies kann Effekte anderer Einflussgrößen überstrahlen (z.B. Haynie E Osgood 2005; Meldrum et al. 2009). Mit Verwendung von Netzwerkdaten zur direkten Messung der Peer-Delinquenz konnten wir nachweisen, dass zwischen Fehlwahrnehmung und eigener Delinquenz ein Zusammenhang besteht. Wird untersucht, ob delinquente Freunde neben den unstrukturierten Freizeitaktivitäten eine Rolle im PCP spielen, so müssen diese in das Regressionsmodell aufgenommen werden. Mit der indirekten Messung kommen wir zu dem Ergebnis, dass es nur die delinquenten Freunde sind, die neben der individuellen kriminellen Neigung einen Einfluss auf die Delinquenz von Jugendlichen haben. Es macht also keinen Unterschied, ob man einen risikoreichen Freizeitstil hat, sondern es kommt lediglich auf die persönlichen Neigungen und die Art der Freunde an. Im Gegensatz dazu sind mit Verwendung der direkten Messung alle drei Prädiktoren von Bedeutung. Die Wechselwirkung von unstrukturierten Freizeitaktivitäten und individueller krimineller Neigung bleibt auch nach Aufnahme der Peer-Delinquenz in das Modell erhalten. Hinzu kommt der Aspekt, dass diese Wechselwirkung umso ausgeprägter wird, je delinquenter die Freunde sind, mit denen man einen risikoreichen Freizeitstil teilt. 
Aus diesen Ergebnissen kann abgeleitet werden, dass es im Rahmen von Untersuchungen zur SAT lohnenswert ist, die Delinquenz von Freunden genauer zu analysieren. Allerdings ist dies nur bedingt mit indirekten Messungen zur Peer-Delinquenz möglich, da diese die Einflüsse anderer Größen überdecken. Sind Netzwerkdaten vorhanden, kann dieses Problem durch die direkte Messung umgangen werden, zudem lassen sich wie bei Haynie (2002) relative Maße zur Messung der Peer-Delinquenz gewinnen. Einschränkend muss jedoch darauf hingewiesen werden, dass in unseren Analysen nur Schüler berücksichtigt wurden, die ihre Freunde hauptsächlich in der Schulklasse hatten. Kiesner, Kerr E Stattin (2004) weisen darauf hin, dass in Bezug auf delinquentes Verhalten besonders die außerschulischen Freunde wichtig sind. Jedoch waren bislang nur wenige Studien in der Lage, Freundesnetzwerke sogar über die Schulgrenzen hinweg zu erheben (Kiesner, Poulin E Nicotra 2003; Kirke 2006; Kerr, Stattin E Kiesner 2007). Zwar wurde das genannte Problem mit unserer Teilstichprobe in gewissem Maße abgefangen, es sollte jedoch davon ausgegangen werden, dass der genannte Befund damit nicht gänzlich eliminiert werden kann und die direkte Messung die Delinquenz der Freunde auch unterschätzen kann. Wie auch Weerman E Smeenk (2005) gehen wir davon aus, dass der wahre Wert des Zusammenhangs zwischen der eigenen Delinquenz und der Delinquenz der Freunde zwischen der direkten und indirekten Messung liegt.

\section{Literatur}

Ai, C. E Norton, E.C. (2003). Interaction terms in logit and probit models. Economics Letters 80, 123-129.

Akers, R.L. (1973). Deviant Behavior: A social learning approach. Belmont.

Akers, R.L. E Jensen, G.F. (2003). Social Learning Theory and the Explanation of Crime: A guide for the new century. New Brunswick/NJ.

Aseltine, R.H., Jr. (1995). A Reconsideration of Parental and Peer Influences on Adolescent Deviance. Journal of Health and Social Behavior 36, 103-121.

Baerveldt, C., Völker, B. E Van Rossem, R. (2008). Revisiting Selection and Influence: An Inquiry into the Friendship Networks of High School Students and Their Association with Delinquency. Canadian Journal of Criminology and Criminal Justice/La Revue canadienne de criminologie et de justice pénale 50, 559-587.

Beier, H. (2014). Peer effects in offending behaviour across contexts: Disentangling selection, opportunity and learning processes. European Journal of Criminology 11, 73-93.

Borgatti, S.P., Carley, K.M. E Krackhardt, D. (2006). On the robustness of centrality measures under conditions of imperfect data. Social Networks 28, 124-136.

Burgess, R.L. E Akers, R.L. (1966). A Differential Association-Reinforcement Theory of Criminal Behavior. Social Problems 14, 128-147.

Burk, W.J., Kerr, M. E Stattin, H. (2008). The co-evolution of early adolescent friendship networks, school involvement, and delinquent behaviors. Revue française de sociologie 49, 499-522.

Burt, R.S. (1987). A note on missing network data in the general social survey. Social Networks 9, 63-73.

Cameron, A.C. E Trivedi, P.K. (2010). Microeconometrics using stata. College Station, TX.

Cillessen, A.H.N. (2009). Sociometric Methods, in: K.H. Rubin, W.M. Bukowski \& B. Laursen (eds.), Handbook of Peer Relationships. Peer interactions and groups. New York, 82-99.

Cohen, L.E. E Felson, M. (1979). Social Change and Crime Rate Trends: A Routine Activity Approach. American Sociological Review 44, 588-608.

Crick, N.R. E Ladd, G.W. (1989). Nominator Attrition: Does It Affect the Accuracy of Children's Sociometric Classifications? Merrill-Palmer Quarterly (1982-) 35, 197-207.

Elliott, D.S., Ageton, S., Huizinga, D., Knowles, B. E Canter, R. (1983). The Prevalence and Incidence of Delinquent Behavior, 1976-1980: National Estimates of Delinquent Behavior by Sex, Race, Social Class and Other Selected Variables. Boulder/CO.

Elliott, D.S., Huizinga, D. E Ageton, S.S. (1985). Explaining Delinquency and Drug Use. Beverly Hills.

Erlhofer, S. (2008). Missing Data in der Netzwerkanalyse, in: C. Stegbauer (Hrsg.), Netzwerkanalyse und Netzwerktheorie. Wiesbaden, 251-260. 
Friemel, T.N. E Knecht, A. (2009). Praktikable vs. tatsächliche Grenzen von sozialen Netzwerken. Eine Diskussion zur Validität von Schulklassen als komplette Netzwerke, in: R. Häußling (Hrsg.), Grenzen von Netzwerken. Wiesbaden, 15-32.

Gardner, M. E Steinberg, L. (2005). Peer Influence on Risk Taking, Risk Preference, and Risky Decision Making in Adolescence and Adulthood: An Experimental Study. Developmental Psychology 41, 625-635.

Giordano, P.C. (2003). Relationships in Adolescence. Annual Review of Sociology 29, 257-281.

Gottfredson, M.R. E Hirschi, T. (1990). A General Theory of Crime. Stanford/CA.

Grasmick, H.G., Tittle, C.R., Bursik, R.J. E Arneklev, B.J. (1993). Testing the Core Empirical Implications of Gottfredson and Hirschi's General Theory of Crime. Journal of Research in Crime and Delinquency 30, 5-29.

Greene, W. (2010). Testing hypotheses about interaction terms in nonlinear models. Economics Letters 107, 291-296.

Handcock, M.S. E Gile, K. (2007). Modeling Social Networks with Sampled or Missing Data. Washington.

Haynie, D.L. (2001). Delinquent Peers Revisited: Does Network Structure Matter? American Journal of Sociology 106, 1013-1057.

Haynie, D.L. (2002). Friendship Networks and Delinquency: The Relative Nature of Peer Delinquency. Journal of Quantitative Criminology 18, 99-134.

Haynie, D.L. E Osgood, D.W. (2005). Reconsidering peers and delinquency: How do peers matter? Social Forces 84, 1109-1130.

Hirschi, T. (1969). Causes of Delinquency. Berkeley u.a.

Hoeben, E. E Weerman, F. (2014). Situational conditions and adolescent offending: Does the impact of unstructured socializing depend on its location? European Journal of Criminology 11, 481-499.

Holgado-Tello, F., Chacón-Moscoso, S., Barbero-García, I. E Vila-Abad, E. (2010). Polychoric versus Pearson correlations in exploratory and confirmatory factor analysis of ordinal variables. Quality \& Quantity 44, 153-166.

Hughes, L.A. E Short, J.F., Jr. (2014). Partying, Cruising, and Hanging in the Streets: Gangs, Routine Activities, and Delinquency and Violence in Chicago, 1959-1962. Journal of Quantitative Crimino$\log 30,415-451$.

Jussim, L. E Osgood, D. W. (1989). Influence and Similarity Among Friends: An Integrative Model Applied to Incarcerated Adolescents. Social Psychology Quarterly 52, 98-112.

Karaca-Mandic, P., Norton, E.C. E Dowd, B. (2012). Interaction Terms in Nonlinear Models. Health Services Research 47, 255-274.

Kerr, M., Stattin, H. E Kiesner, J. (2007). Peers and problem behavior: Have we missed something, in: R.C.M.E. Engels, M. Kerr \& H. Stattin (eds.), Friends, Lovers and Groups: Key relationships in adolescence. Hot topics in developmental research. Chichester, 125-153.

Kiesner, J., Kerr, M. E Stattin, H. (2004). »Very Important Persons in adolescence: Going beyond in-school, single friendships in the study of peer homophily. Journal of Adolescence 27, 545-560.

Kiesner, J., Poulin, F. E Nicotra, E. (2003). Peer Relations Across Contexts: Individual-Network Homophily and Network Inclusion In and After School. Child Development 74, 1328-1343.

Kirke, D.M. (2006). Teenagers and Substance Use: Social networks and peer influence. Basingstoke.

Kossinets, G. (2006). Effects of missing data in social networks. Social Networks 28, 247-268.

Meldrum, R.C., Young, J.T.N. \& Weerman, F.M. (2009). Reconsidering the Effect of Self-Control and Delinquent Peers Implications of Measurement for Theoretical Significance. Journal of Research in Crime and Delinquency 46, 353-376.

Mood, C. (2010). Logistic Regression: Why We Cannot Do What We Think We Can Do, and What We Can Do About It. European Sociological Review 26, 67-82.

Oberwittler, D. (2015). Situative und interaktive Bedingungen, in: W. Melzer, D. Herrmann, U. Sandfuchs, M. Schäfer, W. Schubarth \& P. Daschner (Hrsg.), Handbuch Aggression - Gewalt und Kriminalität bei Kindern und Jugendlichen. Bad Heilbrunn, 88-92.

Oberwittler, D. E Gerstner, D. (2014). Die Modellierung von Interaktionseffekten in Erklärungsmodellen selbstberichteter Delinquenz - Ein empirischer Vergleich von linearer OLS-Regression und negativer Binomialregression anhand der Wechselwirkungen von Risikoorientierung und Scham, in: S. Eifler \& D. Pollich (Hrsg.), Empirische Forschung über Kriminalität. Wiesbaden, 275-301. 
Oberwittler, D., Schwarzenbach, A. E Gerstner, D. (2014). Polizei und Jugendliche in multiethnischen Gesellschaften, Ergebnisse der Schulbefragung 2011 »Lebenslagen und Risiken von Jugendlichen« in Köln und Mannheim. Freiburg im Breisgau.

Osgood, D.W., Wilson, J.K., O'Malley, P.M., Bachman, J.G. E Johnston, L.D. (1996). Routine Activities and Individual Deviant Behavior. American Sociological Review 61, 635-655.

Quetelet, A. (1921 [orig. 1835]). Soziale Physik oder Abhandlung über die Entwicklung der Fähigkeiten der Menschen. Jena.

Raufelder, D. (2010). Soziale Beziehungen in der Schule - Luxus oder Notwendigkeit?, in: A. Ittel, H. Merkens, L. Stecher \& J. Zinnecker (Hrsg.), Jahrbuch Jugendforschung. Wiesbaden, 187-202.

Shaw, C.R. E McKay, H.D. (1931). Report on the Causes of Crime. Washington, DC.

StataCorp. (2013). Stata Statistical Software: Release 13. College Station, TX.

Steglich, C., Snijders, T.A.B. E Pearson, M. (2010). Dynamic Networks and Behavior: Separating Selection from Influence. Sociological Methodology 40, 329-393.

Sutherland, E.H. (1947). Principles of Criminology. Chicago u.a.

Svensson, R. E Oberwittler, D. (2010). It's not the time they spend, it's what they do: The interaction between delinquent friends and unstructured routine activity on delinquency: Findings from two countries. Journal of Criminal Justice 38, 1006-1014.

Svensson, R. E Pawwels, L. (2010). Is a Risky Lifestyle Always »Risky«? The Interaction Between Individual Propensity and Lifestyle Risk in Adolescent Offending: A Test in Two Urban Samples. Crime \& Delinquency 56, 608-626.

Svensson, R., Pauwels, L. E Weerman, F.M. (2010). Does the Effect of Self-Control On Adolescent Offending Vary By Level of Morality? Criminal Justice and Behavior 37, 732-743.

Svensson, Y., Burk, W.J., Stattin, H. E Kerr, M. (2012). Peer selection and influence of delinquent behavior of immigrant and nonimmigrant youths: Does context matter? International Journal of Behavioral Development 36, 178-185.

Thomas, K.J. E McGloin, J.M. (2013). A Dual-Systems Approach for Understanding Differential Susceptibility to Processes of Peer Influence. Criminology 51, 435-474.

Thornberry, T.P., Lizotte, A.J., Krohn, M.D., Farnworth, M. E Jang, S.J. (1994). Delinquent Peers, Beliefs, and Delinquent Behavior: A Longitudinal Test of Interactional Theory. Criminology 32, 47-83.

Tsai, T. E Gill, J. (2013). Interactions in Generalized Linear Models: Theoretical Issues and an Application to Personal Vote-Earning Attributes. Social Sciences 2, 91-113.

Tutz, G. (2010). Regression für Zählvariablen, in: C. Wolf \& H. Best (Hrsg.), Handbuch der sozialwissenschaftlichen Datenanalyse. Wiesbaden, 887-904.

Warr, M. (1996). Organization and Instigation in Delinquent Groups. Criminology 34, 11-37.

Warr, M. (2002). Companions in crime: The social aspects of criminal conduct. Cambridge.

Weerman, F.M. (2011). Delinquent peers in context: A longitudinal network analysis of selection and influence effects. Criminology 49, 253-286.

Weerman, F.M., Bernasco, W., Bruinsma, G.J.N. E Pauwels, L.J.R. (2013). When Is Spending Time With Peers Related to Delinquency? The Importance of Where, What, and With Whom. Crime \& Delinquency (published online before print).

Weerman, F.M. E Smeenk, W.H. (2005). Peer Similarity in Delinquency for Different Types of Friends: A Comparison Using Two Measurement Methods. Criminology 43, 499-524.

Wikström, P.-O.H. (2004). Crime as alternative: Towards a cross-level situational action theory of crime causation, in: J. McCord (ed.), Beyond Empiricism. New Brunswick, London, 1-38.

Wikström, P.-O.H. (2009). Crime propensity, criminogenic exposure and crime involvement in early to mid adolescence. Monatsschrift für Kriminologie und Strafrechtsreform 92, 253-266.

Wikström, P.-O.H. (2010). Situational Action Theory, in: F.T. Cullen \& P. Wilcox (eds.), Encyclopedia of Criminological Theory. Los Angeles u.a., 1000-1008.

Wikström, P.-O.H., Oberwittler, D., Treiber, K. E Hardie, B. (2012). Breaking Rules. The social and situational dynamics of young people's urban crime. Oxford.

Wikström, P.-O.H. E Svensson, R. (2008). Why are English Youths More Violent Than Swedish Youths?: A Comparative Study of the Role of Crime Propensity, Lifestyles and Their Interactions in Two Cities. European Journal of Criminology 5, 309-330.

Wikström, P.-O.H. E Svensson, R. (2010). When does self-control matter? The interaction between morality and self-control in crime causation. European Journal of Criminology 7, 395-410. 
Young, J.T.N., Barnes, J.C., Meldrum, R.C. E Weerman, F.M. (2011). Assessing and explaining misperceptions of peer delinquency. Criminology 49, 599-630.

(Anschr. d. Verf.: Dominik Gerstner, M.A., und PD Dr. Dietrich Oberwittler, Max-Planck-Institut für ausländisches und internationales Strafrecht, Günterstalstr. 73, 79100 Freiburg; d.gerstner@mpicc.de; d.oberwittler@mpicc.de) 